This document is the Accepted Manuscript version of a Published Work that appeared in final form in ACS Sustainable Chemistry and Engineering copyright @ American Chemical Society after peer review and technical editing by the publisher. To access the final edited and published work see https://pubs.acs.org/doi/10.1021/acssuschemeng.8b01998

\title{
Water vapor electrolysis with proton-conducting graphene oxide nanosheets
}

\author{
Tetsuya Kida*†, Yuta Kuwaki, ${ }^{\dagger}$ Azumi Miyamoto, ${ }^{\dagger}$ Nur Laila Hamidah, ${ }^{\dagger}$ Kazuto \\ Hatakeyama, ${ }^{\dagger}$ Armando T. Quitain,${ }^{\dagger}$ Mitsuru Sasaki,${ }^{\dagger}$ Atsushi Urakawa ${ }^{\star}$ \\ $\dagger$ Department of Applied Chemistry and Biochemistry, Kumamoto University, Kurokami, \\ Kumamoto, 860-8555, Japan \\ $\$$ Institute of Chemical Research of Catalonia (ICIQ), The Barcelona Institute of Science and \\ Technology, Tarragona 43007, Spain
}

\section{AUTHOR INFORMATION}

\section{Corresponding Author}

Tetsuya Kida*

Department of Applied Chemistry and Biochemistry, Kumamoto University, Kurokami,

Kumamoto, 860-8555, Japan

E-mail: tetsuya@kumamoto-u.ac.jp

p. 1 


\begin{abstract}
Hydrogen production by membrane water electrolysis has attracted tremendous attention because of its benefits, which include easy separation of hydrogen and oxygen, no carbon emissions, and the possibility to store hydrogen fuel as an electricity source. Here, we study water vapor electrolysis using a proton-conducting membrane comprising graphene oxide (GO) nanosheets. The GO membrane shows good through-plane proton conductivity, as confirmed by concentrationcell measurements, complex impedance spectroscopy, and hydrogen pumping experiments. The results also confirm that most carriers in the GO membrane are protons. The GO membrane fitted with $\mathrm{Pt} / \mathrm{C}$ and $\mathrm{IrO}_{2}-\mathrm{Al}_{2} \mathrm{O}_{3}$ as the cathode and the anode, respectively, efficiently electrolyzes humidified air to produce hydrogen and oxygen at room temperature, which indicates bright prospects for this carbon-based electrochemical device.
\end{abstract}

Keywords: Graphene oxide; water electrolysis; proton conductor; membrane; $\mathrm{IrO}_{2}$

p. 2 


\section{INTRODUCTION}

Graphene oxide (GO) has become an intensive research target in electrochemistry because of its tunable physical and chemical properties, low material cost, good stability, and versatility. ${ }^{[1-4]}$ Numerous applications for GO have been proposed in various fields; one promising property of GO is its proton conductivity, [5,6] which allows for the development of carbon-based electrochemical devices such as fuel cells, ${ }^{[7-9]}$ capacitors, ${ }^{[10]}$ gas sensors, ${ }^{[11]}$ electrocatalysts, ${ }^{[12-}$ ${ }^{13]}$ and transistors. ${ }^{[14-15]}$

GO is a carbon nanosheet with oxygen functional groups such as hydroxyl, epoxy, carbonyl, and carboxyl groups. A membrane made by stacking GO nanosheets shows a good proton conductivity because of the presence of oxygen functional groups. There is a clear correlation between proton conductivity and the amount of epoxy groups, which indicates that epoxy groups on GO serve as proton hopping sites. ${ }^{[6,16]}$ The interlayer water assists the proton diffusion. Incorporating sulfonate ions into a GO membrane also effectively improved proton conductivity by introducing more protons in the interlayer. ${ }^{[17]}$ The in-plane proton conductivity can be explained in terms of the interlayer diffusion via hopping on oxygen functional groups, whereas the through-plane diffusion is probably regulated by defects (nanopores or nanocavities) in GO nanosheets or spaces between each stacked nanosheet. ${ }^{[6]}$

Proton conduction in solids is important for reacting and separating hydrogen. ${ }^{[18,19]}$ Currently, water electrolysis using a proton-conducting membrane has attracted much attention because it

\section{p. 3}


generates pure hydrogen from water without producing unwanted products such as carbon dioxide or hydrocarbons. ${ }^{[20]}$ Another merit of the membrane reactor is that hydrogen can easily be separated from the oxygen that is simultaneously formed by water electrolysis. Steam electrolysis is also possible using proton-conducting membranes. ${ }^{[21]}$ For these applications, a solid electrolyte membrane plays is necessary to separate the electrodes (anode and cathode) and mediate proton transfer to maintain the reaction. Polymers such as perfluoro-3,6-dioxa-4-methyl-7-octenesulfonic acid (Nafion, Du Pont) and ceramics such as $\mathrm{SrZrO}_{3}$ and $\mathrm{SrCeO}_{3}$ have often been used as membrane materials. Nafion has several advantages over other materials, including high proton conductivity at room temperature (approximately $10^{-2} \mathrm{Scm}^{-1}$ ), excellent thermal and mechanical stability, and flexibility. However, its high cost has spurred the development of cheaper alternative membranes.

In this study, we focused on using proton-conducting GO to electrochemically produce hydrogen from humid air. GO is stable at room temperature, cheap, and environmentally friendly. The development of a water electrolysis system based on non-toxic and abundant carbon materials is important for a sustainable society that relies on renewable energy and materials. Using GO's ultra-thin nature, the development of an ultra-small electrolysis device is also expected. Here, we demonstrate that a membrane comprising GO nanosheets fitted with electrocatalysts can potentially electrolyze water vapor into hydrogen and oxygen at room temperature.

\section{EXPERIMENTAL}

\section{Synthesis of GO nanosheets and membrane fabrication}

p. 4 
GO was prepared from graphite powder using a modified Hummers' method according to our previous report. ${ }^{11)}$ Graphite (2.0 g) was added to concentrated sulfuric acid (92 mL) while stirring, and then sodium nitrate $(2.0 \mathrm{~g})$ was added. The mixture was cooled in an ice bath for 30 minutes. Subsequently, potassium permanganate $(10 \mathrm{~g})$ was added slowly into the mixture. The reaction system was then slowly transferred to a water bath and heated at $35^{\circ} \mathrm{C}$ for 40 minutes. Distilled water $(92 \mathrm{~mL})$ was slowly added to the solution, which was stirred for another 5 hours in an oil bath at $95^{\circ} \mathrm{C}$. Next, distilled water $(200 \mathrm{~mL})$ and hydrogen peroxide were added until the color of the solution changed from brown to yellow. After cooling, the product was recovered by centrifugation at 3,000 rpm for 5 minutes. The recovered precipitate was washed with an aqueous $\mathrm{HCl}$ solution (5\%) three times to remove metal ions. The obtained solid was dispersed in distilled water, followed by ultrasonication for 4-6 hours. The resulting dispersion was centrifuged at 10,000 rpm for 30 minutes. Finally, the supernatant was separated to obtain a colloidal suspension containing GO nanosheets. A self-supporting membrane was fabricated on two filter membranes stacked together using the GO suspension $(3.5 \mathrm{mg} / \mathrm{mL})$ by vacuum filtration. The membrane thickness was $180 \mu \mathrm{m}$.

\section{Fabricating and electrochemically testing a device}

The GO membrane was coated with a paste containing Pt (46.3\%)/carbon (TEC10E50E, Tanaka Holdings Co., Ltd.) in isopropanol. The Pt/C layers on both sides of the membrane were dried at room temperature in ambient air to form the anode and the cathode. The GO membrane with $\mathrm{Pt} / \mathrm{C}$ layers was tightly sandwiched with two glass tubes with gas inlets and outlets. Pt meshes were inserted between the membrane and the glass tubes as electron collectors for the anode and

\section{p. 5}


cathode; these Pt meshes were connected to an electrometer (R8240, Advantest) and a D.C. generator (TR6142, Advantest). The device was put in an incubator and heated at $40-80^{\circ} \mathrm{C}$.

This device was used for concentration-cell measurements, in which hydrogen (1-5\%) in humified Ar (90\% relative humidity [RH]) was introduced to the anode and the cathode. Humid gases were prepared by bubbling synthetic air through distilled water, and the humidity was checked with a humidity sensor. The total flow rate of the sample gases was controlled by mass flow controllers to $100 \mathrm{~mL} / \mathrm{min}$. The electromotive force (EMF) generated between the anode and the cathode was measured with an electrometer. For hydrogen pumping, hydrogen (5\%) in dry Ar was introduced to the anode side, while humified $\operatorname{Ar}(90 \% \mathrm{RH})$ was introduced to the cathode side. For water vapor electrolysis, humified $\operatorname{Ar}(90 \% \mathrm{RH})$ was introduced to the anode side, while dry Ar was introduced to the cathode side. Any hydrogen and oxygen that evolved at the cathode and the anode, respectively, were detected by gas chromatography (GC-8A, Shimadzu).

\section{Synthesis of $\mathrm{IrO}_{2} / \mathrm{Al}_{2} \mathrm{O}_{3}$ and its use in the anode}

$\mathrm{IrO}_{2}(30 \mathrm{wt} \%)-\mathrm{Al}_{2} \mathrm{O}_{3}$ was synthesized using a solution combustion method reported in literature. ${ }^{22)}$ Iridium chloride $\left(\mathrm{IrCl}_{3} \cdot 2.65 \mathrm{H}_{2} \mathrm{O}: 100 \mathrm{mg}\right)$, aluminum nitrate $\left(\mathrm{Al}\left(\mathrm{NO}_{3}\right)_{3} \cdot 9 \mathrm{H}_{2} \mathrm{O}: 455\right.$ $\mathrm{mg}$ ), and glycine $(227 \mathrm{mg}$ ) were dissolved in distilled water $(35 \mathrm{~mL})$ under stirring for 30 minutes. The solution was heated in an alumina crucible at $80^{\circ} \mathrm{C}$ with a hot-plate to form a viscous gel. The gel was heated at $400^{\circ} \mathrm{C}$ in a furnace for 2 hours.

The obtained powder was coated on the GO membrane as the anode using the above method with an ink containing $\mathrm{IrO}_{2}-\mathrm{Al}_{2} \mathrm{O}_{3}$ in isopropanol. For comparison, a commercial paste containing colloidal graphite (G7711, EMJapan Co., Ltd.) was also coated on a GO membrane and used as the anode.

p. 6 


\section{Material characterization}

The fabricated GO membrane was analyzed by X-ray diffraction (XRD) using $\mathrm{Cu}$ K $\alpha$ radiation (MiniFlex600, Rigaku). The presence of oxygen functional groups on GO was analyzed using Fourier transform infrared (FT-IR) spectroscopy (FTIR4100, JASCO) and X-ray photoemission spectroscopy (PHI 1600, PerkinElmer). The proton conductivity of the GO membrane was examined using complex impedance spectroscopy. Pt electrodes were sputtered onto both sides of the membrane. The membrane was put in an incubator and its impedance was measured in air under humid conditions (2-90\% RH) with an impedance analyzer (1260, Solartron).

\section{RESULTS AND DISCUSSION}

A GO membrane was fabricated using the scheme shown in Figure 1 (a). The oxidation and exfoliation of graphite produced a stable suspension containing GO nanosheets in water. The colloidal size of GO nanosheets in water was in the range of 70 to $800 \mathrm{~nm}$, as determined by dynamic light scattering ${ }^{[11]}$. Atomic force microscopy clearly showed the formation of 1.2-nmthick nanosheets, as shown in Figure S1 (Supporting Information). The presence of oxygen functional groups was confirmed using FT-IR and X-ray photoelectron spectroscopy. The representative FT-IR spectrum of the GO membrane is shown in Figure S2. The oxygen content in GO was $57 \mathrm{wt} \%$. The obtained GO nanosheet suspension in water $(3.5 \mathrm{mg} / \mathrm{mL})$ was filtered on a filter membrane. Stacked nanosheets were separated from the filter membrane to use them as a self-standing membrane (180 $\mu \mathrm{m}$ thick). Figure 1 (b) depicts the structure of a device comprising the GO membrane attached with a layer of $\mathrm{Pt}(40 \mathrm{wt} \%) / \mathrm{C}$ on the both membrane sides. DC voltages 
were applied to the electrodes and the fluxes of hydrogen and oxygen produced at the cathode and the anode, respectively, were measured using gas chromatography. The RH of sample gases was controlled by bubbling through distilled water.
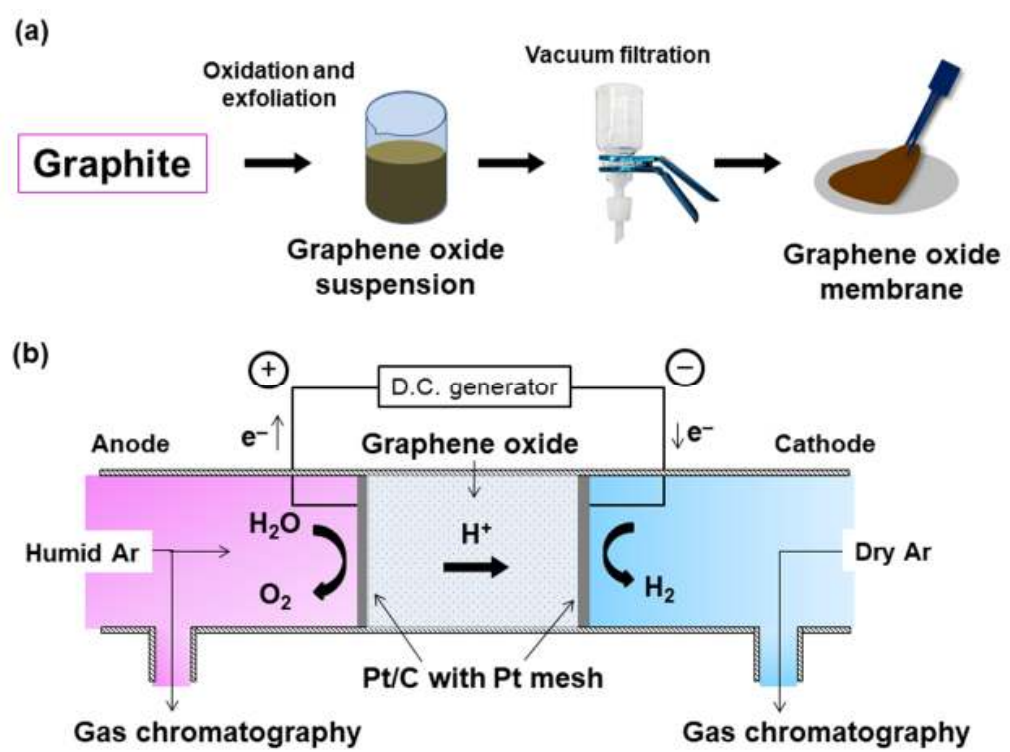

Figure 1. (a) Fabrication process of a GO membrane comprising GO nanosheets. (b) Structure of a GO device for concentration-cell measurements, hydrogen pumping, and water vapor electrolysis. For water vapor electrolysis, humid $\operatorname{Ar}(90 \% \mathrm{RH})$ was introduced to the anode, while dry $\mathrm{Ar}$ was introduced to the cathode as a sweep gas. For concentration-cell measurements, humified $\mathrm{H}_{2}$ in $\mathrm{Ar}(90 \% \mathrm{RH})$ was introduced to both sides. For hydrogen pumping, $\mathrm{H}_{2}$ in dry $\mathrm{Ar}$ was introduced to the anode, while humid $\operatorname{Ar}(90 \% \mathrm{RH})$ was introduced to the cathode.

First, the transfer number $(t)$ of proton conduction in a GO membrane was determined by concentration-cell measurements using the cell shown in Figure 1. The EMF generated between the anode and the cathode was measured upon changing the hydrogen concentration at the anode p. 8 
side. Figure 2 (a) shows the time dependence of the EMF response of the GO membrane fitted with $\mathrm{Pt} / \mathrm{C}$ electrodes to changes in hydrogen concentration at room temperature. The GO membrane showed good EMF responses to hydrogen, which suggests the occurrence of the equilibrium reaction of hydrogen and protons with a reaction number $(n)$ of $2\left(\mathrm{H}_{2}=2 \mathrm{H}^{+}+2 \mathrm{e}^{-}\right)$. Figure 2 (b) shows the dependence of EMF on hydrogen concentration in Ar. The EMF was linear to the logarithm of hydrogen concentration. The slope of the linear curve was $28.2 \mathrm{mV} / \mathrm{decade}$, in good agreement with the expected result from the following Nernst's equation when $n=2(29.5$ $\mathrm{mV} /$ decade):

$$
\mathrm{EMF}=\frac{R T}{n F} t \ln \frac{p_{\mathrm{H} 2}^{\prime \prime}}{p_{\mathrm{H} 2}^{\prime}}
$$

where $R$ is the gas constant, $T$ is the absolute temperature, $F$ is the Faraday constant, and $P_{\mathrm{H} 2}$ " and $P_{\mathrm{H} 2}$ are the hydrogen partial pressures at the anode and cathode, respectively. The proton transport number $(t)$ calculated from the slope was close to unity (0.956). A slight deviation was observed at higher $\ln \left(P_{\mathrm{H} 2}{ }^{\prime \prime} / P_{\mathrm{H} 2}\right)$, suggesting that the GO membrane also has little electrical conductivity. However, at higher temperatures, $t$ was always below 1.0, as shown in Figure 2 (c). Indeed, no response to hydrogen was observed at $80^{\circ} \mathrm{C}$. To reveal the reason for the decrease in $t$, the interlayer distances of GO membranes after annealing at different temperatures were examined by XRD. The XRD peak shifted to higher angles after annealing, as shown in Figure S3. The interlayer distance, calculated from the peak angles, changed from 13.32 to $3.47 \AA$ after annealing at $100^{\circ} \mathrm{C}$, as shown in Table S1. The results suggest that oxygen functional groups desorbed from GO nanosheets at higher temperatures. The significant decrease in the interlayer distance above $100^{\circ} \mathrm{C}$ was probably due to the removal of water from the interlayer. The appearance of electron 
conductivity must be due to the removal of oxygen functional groups at higher temperatures. The resulting formation of $\mathrm{sp}^{2}$-bonding carbon regions induced the electronic conduction in the GO membrane, leading to the decrease in $t$.

The through-plane proton conductivity of the GO membrane at $35 \% \mathrm{RH}$ was measured at different temperatures using complex impedance spectroscopy (Figure 2 [d]). The impedance results were fitted with an equivalent circuit that comprises the bulk resistance, the interface resistance, and the interface capacitance (Figure S4). The GO showed a through-plane proton conductivity of $16 \mathrm{mS} / \mathrm{cm}(80 \% \mathrm{RH})$ at room temperature, which is comparable to that for Nafion (approximately $10-20 \mathrm{mS} / \mathrm{cm}$ ). The proton conductivity was higher at $40^{\circ} \mathrm{C}$ because of the thermal activation of proton diffusion. The interlayer water also assisted the proton diffusion in the membrane. However, it sharply decreased at $60^{\circ} \mathrm{C}$ because of the partial removal of oxygen functional groups. 

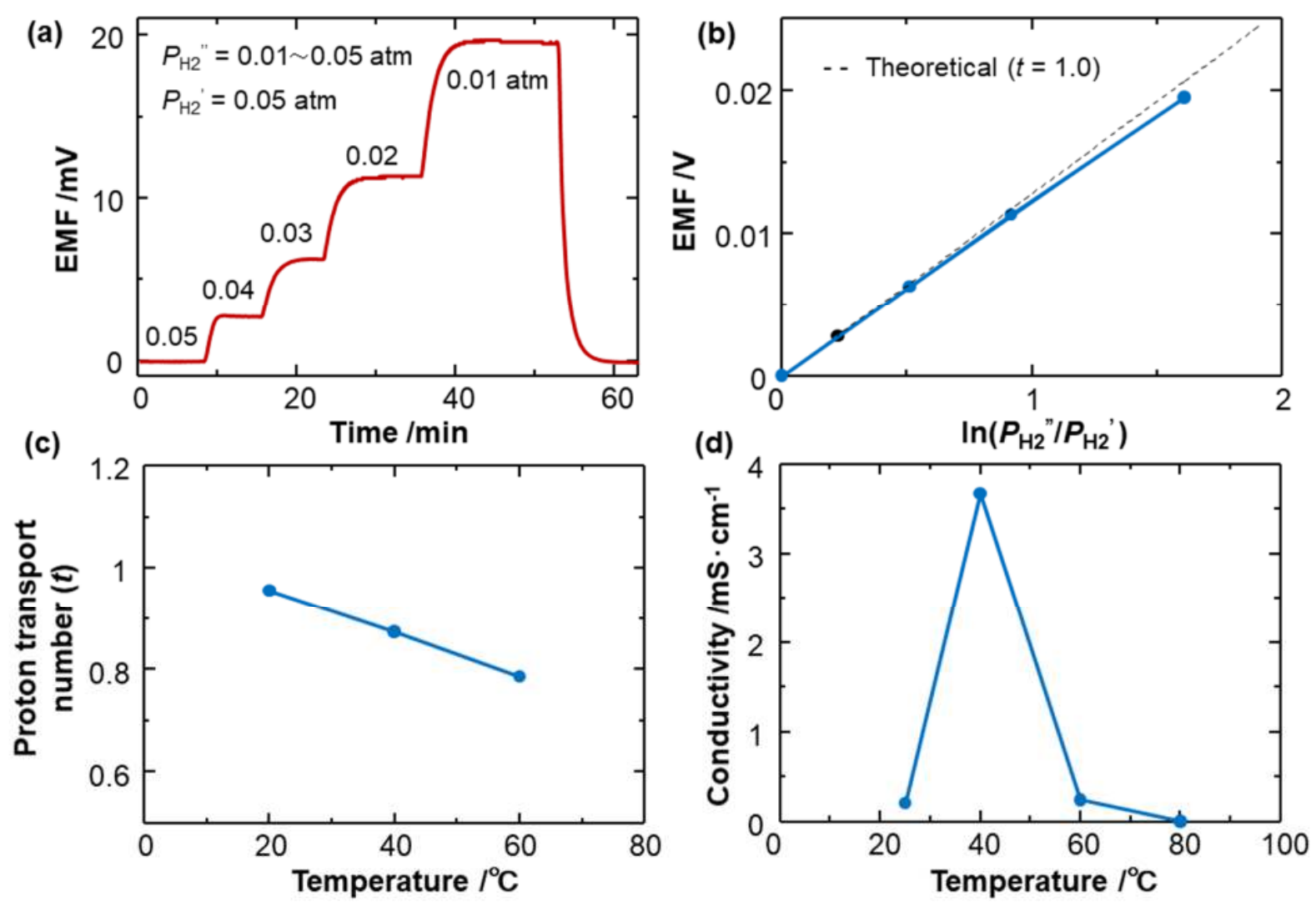

Figure 2. (a) Time-dependent EMF response of the GO device upon changes in hydrogen concentration in Ar at room temperature. (b) Dependence of EMF on hydrogen concentration. (c) Dependence of proton transport number $(t)$ on temperature. (d) Dependence of through-plane proton conductivity at $35 \% \mathrm{RH}$ on temperature.

The results of concentration-cell measurements and complex impedance spectroscopy clearly show that the GO membrane has a good proton conductivity at room temperature. To confirm proton conduction in the GO membrane, we also used it for hydrogen pumping using $\mathrm{Pt}$ (40 $\mathrm{wt} \%) / \mathrm{C}$ as the anode and the cathode. Voltages ranging from 1.0 to $2.5 \mathrm{~V}$ were applied to the electrodes and hydrogen produced at the cathode side was detected by gas chromatography. 
Hydrogen in dry Ar was introduced to the anode side, while humified $\operatorname{Ar}(90 \% \mathrm{RH})$ was introduced to the cathode side to avoid loss of water in the interlayer of the GO membrane. Figure 3 (a) shows the hydrogen evolution flux at the cathode side at room temperature. The hydrogen evolution rate linearly increased with increasing current density. The correlation between the hydrogen flux and the current density is in good agreement with that expected from Faraday's law, which indicates that hydrogen was oxidized to form protons at the anode $\left(\mathrm{H}_{2} \rightarrow 2 \mathrm{H}^{+}+2 \mathrm{e}^{-}\right)$and protons were reduced at the cathode to produce hydrogen $\left(2 \mathrm{H}^{+}+2 \mathrm{e}^{-} \rightarrow \mathrm{H}_{2}\right)$. The results again confirm the proton diffusion in the GO membrane that was created by stacking GO nanosheets.

Next, the GO membrane was used for water vapor electrolysis. The performance of the GO membrane was first examined using Pt mesh electrodes as the anode and the cathode. A Nafion membrane $(178 \mu \mathrm{m}$ thick) that was sandwiched with Pt mesh electrodes was also used for comparison. In the measurements, humified $\operatorname{Ar}(90 \% \mathrm{RH})$ was introduced to the anode side. The hydrogen flux at the cathode as a function of current density is shown in Figure S5. The hydrogen generation was confirmed when applying voltages more than $3 \mathrm{~V}$, but the hydrogen fluxes were lower than the theoretical values expected using Faraday's law. Nevertheless, the GO membrane showed hydrogen fluxes that approached theoretical values at 5 and $8 \mathrm{~V}$ : the fluxes were comparable to those obtained with a Nafion membrane attached with Pt mesh electrodes, which suggested the potential use of GO as an alternative to Nafion to produce hydrogen from water.

To improve performance, $\mathrm{Pt}(40 \mathrm{wt} \%) / \mathrm{C}$ was coated on the GO membrane as the anode and the cathode. The Pt/C-coated GO membrane was then sandwiched with Pt meshes and tested for its performance in water vapor electrolysis. Using $\mathrm{Pt} / \mathrm{C}$ electrodes in place of $\mathrm{Pt}$ mesh electrodes, current density was much improved, as shown in Figure 3 (b). The significant performance enhancement likely originated from the high electrocatalytic activity of Pt nanoparticles in water

\section{p. 12}


electrolysis. The formation of oxygen was confirmed on the anode side. The correlation between the hydrogen-oxygen evolution rates and current density was consistent with the theoretical one. The results indicate that water vapor was oxidized at the anode to produce oxygen $\left(\mathrm{H}_{2} \mathrm{O} \rightarrow 2 \mathrm{H}^{+}+\right.$ $\left.\mathrm{O}_{2}+2 \mathrm{e}^{-}\right)$and the resulting protons were reduced at the cathode to produce hydrogen $\left(2 \mathrm{H}^{+}+2 \mathrm{e}^{-} \rightarrow\right.$ $\mathrm{H}_{2}$ ). However, deviations from theoretical values were observed, particularly at higher current densities. It is probable that the water oxidation is limited because of insufficient $\mathrm{Pt} / \mathrm{C}$ electrode activity, although $\mathrm{Pt} / \mathrm{C}$ has a good electrochemical activity for proton reduction, as shown in Figure 3 (a). The results suggest that the rate-limiting step in water vapor electrolysis for the GO device is water oxidation at the anode, as frequently reported in many systems. ${ }^{[23,24]}$

The performance of the GO membrane at higher temperatures was also examined. Figure 3 (c) shows the hydrogen evolution rate as a function of current density at $20-60^{\circ} \mathrm{C}$. The hydrogen evolution rate decreased as the operating temperature increased. In addition, no hydrogen evolved at $80^{\circ} \mathrm{C}$, even at higher voltages. As shown above, the proton transport number largely deviated from unity at 40 and $60^{\circ} \mathrm{C}$, although the proton conductivity was improved at $40^{\circ} \mathrm{C}$. It is thus obvious that the appearance of electron conduction in the GO membrane degraded the performance of water vapor electrolysis. Steam electrolysis is preferably operated at more than $100^{\circ} \mathrm{C}$ because of the enhanced electrochemical efficiency. ${ }^{[21]}$ However, high temperature steam electrolysis requires energy to heat the system and vaporize water. Conversely, low temperatures may be more suited for unprocessed natural water vapor electrolysis because water concentration can be enhanced at the electrode because of condensation. Another merit is the possibility to significantly downsize the electrolyzer using the nanosheet nature of GO. This would lead to a battery-operated, compact, and portable device that can produce hydrogen from humid air anywhere and anytime. 
Finally, different materials were employed as the anode in place of $\mathrm{Pt} / \mathrm{C}$ to improve the performance of the $\mathrm{GO}$ device. We focused on $\mathrm{IrO}_{2}-\mathrm{Al}_{2} \mathrm{O}_{3}$ nanoparticles that were synthesized by solution combustion synthesis using glycine as a fuel. ${ }^{[22]}$ Highly-dispersed $\mathrm{IrO}_{2}$ nanoparticles within nanoparticulate $\mathrm{Al}_{2} \mathrm{O}_{3}$ matrices (both 1-2 nm in diameter) show an excellent electrochemical activity in water oxidation using a Nafion membrane. Figure 3 (d) shows the hydrogen evolution rate as a function of current density at room temperature for a GO membrane attached with $\mathrm{Pt}(40 \mathrm{wt} \%) / \mathrm{C}$ and $\mathrm{IrO}_{2}(30 \mathrm{wt} \%)-\mathrm{Al}_{2} \mathrm{O}_{3}$ as the cathode and the anode, respectively. The device with $\mathrm{IrO}_{2}-\mathrm{A}_{2} \mathrm{O}_{3}$ at the anode efficiently evolved theoretical amounts of hydrogen expected from Faraday's law with an increasing current density. The evolution of theoretical amounts of oxygen was also confirmed, as shown in Figure S6. In contrast, the amounts of hydrogen for devices using $\mathrm{Pt} / \mathrm{C}$ and colloidal graphite were less than the theoretical amounts. It is likely that the activation of the water vapor oxidation by the $\mathrm{IrO}_{2}$ nanoparticles enhanced the device's performance. However, the performance of the GO device remains lower than that of the Nafion device, which showed higher current density at the same applied voltages, as shown in Figure 3 (d) and Figure S7. The lower proton conductivity in GO or insufficient electrochemical activity at the $\mathrm{GO} /$ electrode interface may be the reason for the lower performance. We believe that controlling the membrane thickness and morphology of the electrode catalysts would further improve performance.

p. 14 

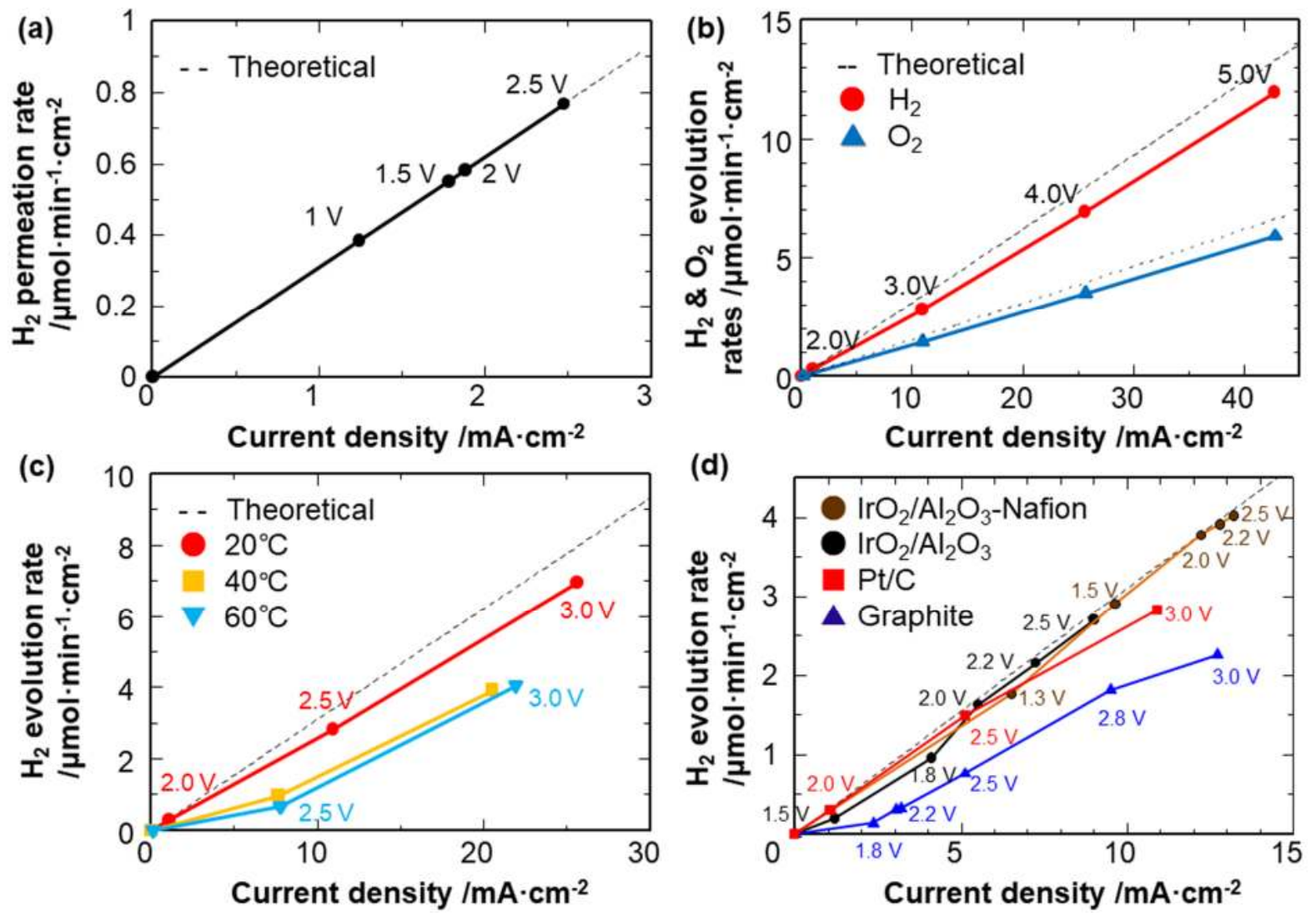

Figure 3 (a) Hydrogen evolution rate vs. current density for the GO device in hydrogen pumping.

(b) Hydrogen and oxygen evolution rates vs. current density in water vapor electrolysis at room temperature. (c) Hydrogen evolution rate vs. current density in water vapor electrolysis at different temperatures. For these measurements, Pt/C electrodes were used as the anode and cathode. (d) Hydrogen evolution rate vs. current density in water vapor electrolysis at room temperature for GO devices using $\mathrm{IrO}_{2}-\mathrm{Al}_{2} \mathrm{O}_{3}$ and colloidal graphite as the anode. The performance of a Nafion device using $\mathrm{IrO}_{2}-\mathrm{Al}_{2} \mathrm{O}_{3}$ as the anode was also compared. For the above tests, $\mathrm{Pt} / \mathrm{C}$ electrodes were used as the cathode. 
The obtained results indicate a bright prospect of this carbon-based electrochemical device to produce hydrogen from humid air at room temperature. However, it is necessary to tune the composition and morphology of the electrode and increase the proton conductivity in the GO membrane to improve performance. The noticeable feature of the GO membrane is its easy fabrication process, whereby filtration of GO nanosheets using a filter membrane produces a dense GO membrane (180 $\mu \mathrm{m}$ thick). The gas tightness of the GO membrane was checked by a helium permeation test, which showed that it was free from pinholes and cracks. However, each GO nanosheet may possibly have nanodefects that form during oxidation of starting graphite, allowing protons to diffuse through defects in the through-plane direction of the membrane. Nano spaces between each nanosheet are also probable diffusion paths for protons. However, the diffusion in the in-plain direction should be assisted by oxygen functional groups. Thus, controlling the lateral size of GO nanosheets and their defect size by repeated oxidation possibly assists the diffusion rate of protons, thereby improving the hydrogen permeation rate. In this sense, synthesizing GO nanosheets by other reported methods such as Tour's, ${ }^{[25]}$, Sun's ${ }^{[26]}$ and Peng's ${ }^{[27]}$ methods would change the oxidation and defect states. Thus, different synthesis routes are worth testing to improve the performance of the GO device and study the fundamental mechanism of proton transport in GO. The intercalation of additives such as sulfate ions and polyoxometalates likely improves the proton conductivity and stability, as reported in the literature. ${ }^{[17,28]}$ The thermal stability would also be improved by synthesizing more oxidized GO, ${ }^{[26]}$ hybridizations of GO with polymers, ${ }^{[29,}$ ${ }^{30]}$ ionic liquids, ${ }^{[31]}$ and so on. Therefore, we believe that it is straightforward to further upgrade the performance of the GO device with the above-mentioned methods.

\section{CONCLUSIONS}

p. 16 
GO nanosheets synthesized by a modified Hummers' method were assembled to form a selfstanding membrane by a simple filtration method. The membrane showed good proton conductivity at room temperature, as revealed by concentration-cell measurements and complex impedance spectroscopy. Proton conduction in the GO membrane was confirmed by hydrogen pumping experiments. The results also confirm that most carriers are protons in the GO membrane. The membrane was applied to water vapor electrolysis at room temperature. It showed good hydrogen and oxygen evolution rates that are close to theoretical values. The results demonstrate the promising capability of GO as an alternative to Nafion in producing hydrogen from water vapor by electrolysis. The GO's nanosheet nature allows for the ultra-miniaturization of an electrolyzer that can produce hydrogen from humid air, which would offer a new hydrogen-producing technique.

Synopsis. Proton-conducting carbon-based membranes were successfully used to electrochemically produce hydrogen from humid air.

\section{ASSOCIATED CONTENT}

Supporting Information. Representative atomic force microscopy images, Fourier transform infrared spectrum, X-ray diffraction patterns at different annealing temperatures, and Cole-Cole plots of the membranes. Control experiment results using a Nafion membrane. The Supporting Information is available free of charge on the ACS Publications website.

\section{p. 17}




\section{ACKNOWLEDGMENTS}

This work was supported in part by the JST e-ASIA collaborative research project on functional materials and by a Grant-in-Aid for the Promotion of Joint International Research (15KK0189) and Grant-in-Aid for Scientific Research (B) (18H01835) from the Ministry of Education, Culture, Sports, Science and Technology of Japan. T.K. thanks Joan Giner of ICIQ for his support to synthesize $\mathrm{IrO}_{2}-\mathrm{Al}_{2} \mathrm{O}_{3}$. A.U. thanks the Generalitat de Catalunya for financial support through the CERCA Programme and recognition (2014 SGR 893) and MINECO (CTQ2016-75499-R (FEDER-UE)) for financial support and support through Severo Ochoa Excellence Accreditation 2014-2018 (SEV-2013-0319).

\section{REFERENCES}

(1) Kuila, T.; Mishra, A.K.; Khanra, P.; Kim, N.H.; Lee, J.H. Recent advances in the efficient reduction of graphene oxide and its application as energy storage electrode materials. Nanoscale 2013, 5 (1), 52-71, DOI 10.1039/C2NR32703A.

(2) Bonaccorso, F.; Colombo, L.; Yu, G.; Stoller, M.; Tozzini, V.; Ferrari, A.C; Ruoff, R.S.; Pellegrini, V. 2D materials. Graphene, related two-dimensional crystals, and hybrid 
systems for energy conversion and storage. Science 2015, 347 (6217), 1246501, DOI $10.1126 /$ science. 1246501 .

(3) Wu, S.; He, Q.; Tan, C.; Wang, Y.; Zhang, H. Graphene-based electrochemical sensors. Small 2013, 9 (8), 1160-1172, DOI 10.1002/smll.201202896.

(4) Eda, G.; Chhowalla, M. Chemically derived graphene oxide: towards large-area thin-film electronics and optoelectronics. Adv. Mater. 2010, 22 (22), 2392-2415, DOI 10.1002/adma.200903689.

(5) Karim M.R.; Hatakeyama K.; Matsui T.; Takehira H.; Taniguchi T.; Koinuma M.; Matsumoto Y.; Akutagawa, T.; Nakamura, T.; Noro, S.-I.; Yamada, T.; Kitagawa, H.; Hayami S. Graphene oxide nanosheet with high proton conductivity. J. Am. Chem. Soc. 2013, 135 (22), 8097-8100, DOI 10.1021/ja401060q.

(6) Hatakeyama, K.; Karim, M.R.; Ogata, C.; Tateishi, H.; Funatsu, A.; Taniguchi, T.; Koinuma, M.; Hayami, S.; Matsumoto, Y. Proton conductivities of graphene oxide nanosheets: Single, multilayer, and modified nanosheets. Angew. Chem. 2014, 53 (27), 6997-7000, DOI 10.1002/anie.201309931.

(7) Tateishi, H.; Hatakeyama, K.; Ogata, C.; Gezuhara, K.; Kuroda, J.; Funatsu, A.; Koinuma, M.; Taniguchi, T.; Hayami, S.; Matsumoto, Y. Graphene oxide fuel cell. J. Electrochem. Soc. 2013, 160 (11), F1175-F1178, DOI 10.1149/2.008311jes.

(8) Zarrin, H.; Higgins, D.; Jun, Y.; Chen, Z.; Fowler, M. Functionalized graphene oxide nanocomposite membrane for low humidity and high temperature proton exchange membrane fuel cells. J. Phys. Chem. C 2011, 115 (42), 20774-20781, DOI 10.1021/jp204610j.

(9) Bayer, T.; Selyanchyn, R.; Fujikawa, S.; Sasaki, K.; Lyth, S.M. Spray-painted graphene oxide membrane fuel cells. J. Memb. Sci. 2017, 541 (1), 347-357, DOI 10.1016/j.memsci.2017.07.012. 
(10)Ogata, C.; Kurogi, R.; Hatakeyama, K.; Taniguchi, T.; Koinuma, M.; Matsumoto, Y. Allgraphene oxide device with tunable supercapacitor and battery behaviour by the working voltage. Chem. Commun. 2016, 52 (20), 3919-3922, DOI 10.1039/C5CC09575A.

(11)Miyamoto A.; Kuwaki Y.; Sano, T.; Hatakeyama, K.; Quitain, A.; Sasaki, M.; Kida T. Solid electrolyte gas sensor based on a proton-conducting graphene oxide membrane. ACS Omega 2017, 2 (6), 2994-3001, DOI 10.1021/acsomega.7b00239.

(12)Chen, X.; Wu, G.; Chen, J.; Chen, Z.; Xie, Z.; Wang, X. Synthesis of “clean" and welldispersive Pd nanoparticles with excellent electrocatalytic property on graphene oxide. $J$. Am. Chem. Soc., 2011, 133 (11), 3693-3695, DOI: 10.1021/ja110313d.

(13)Zhang, H.; Cai, K.; Wang, P.; Huang, Z.; Liu, J.; Wu, L.; Foda, M. F.; Han, H. Graphene oxide as a stabilizer for "clean" synthesis of high-performance Pd-based nanotubes electrocatalysts. ACS Sustainable Chem. Eng. 2017, 5 (6), 5191-5199, DOI: 10.1021/acssuschemeng. 7b00544.

(14)Tsuchiya, T.; Terabe, K.; Aono, M. In situ and non-volatile bandgap tuning of multilayer graphene oxide in an all-solid-state electric double-layer transistor. Adv. Mater. 2014, 26 (7), 1087-1091, DOI 10.1002/adma.201304770.

(15)Wan, C.J.; Zhu, L.Q.; Liu, Y.H.; Feng, P.; Liu, Z.P.; Cao, H.L.; Xiao, P.; Shi, Y., Wan, Q. Proton-conducting graphene oxide-coupled neuron transistors for brain-inspired cognitive systems. Adv. Mater. 2016, 28 (18), 3557-3563, DOI 10.1002/adma.201505898.

(16)Ikeda, Y.; Karim, M.R.; Takehira, H.; Matsui, T.; Taniguchi, T.; Koinuma, M.; Matsumoto, Y.; Hayami, S. Proton conductivity of graphene oxide hybrids with covalently functionalized alkylamines. Chem. Lett. 2013, 42 (11), 1412-1414, DOI 10.1246/cl.130606.

(17)Hatakeyama, K.; Razaul Karim, M.; Ogata, C.; Tateishi, H.; Taniguchi, T.; Koinuma, M.; Hayami, S.; Matsumoto, Y. Optimization of proton conductivity in graphene oxide by filling sulfate ions. Chem. Commun. 2014, 50 (93), 14527-14530, DOI 10.1039/C4CC07273A. 
(18)Tao, Z.; Yan, L.; Qiao, J.; Wang, B.; Zhang, L., Zhang; J. A review of advanced protonconducting materials for hydrogen separation. Prog. Mater. Sci. 2015, 74, 1-50, DOI 10.1016/j.pmatsci.2015.04.002.

(19)Malavasi, L.; Fisher, C.A.J.; Islam, M.S. Oxide-ion and proton conducting electrolyte materials for clean energy applications: Structural and mechanistic features. Chem. Soc. Rev. 2010, 39 (11), 4370-4387, DOI 10.1039/B915141A.

(20)Goñi-Urtiaga, A.; Presvytes, D.; Scott, K. Solid acids as electrolyte materials for proton exchange membrane (PEM) electrolysis: Review. Int. J. Hydro. Ener. 2012, 37 (4), 33583372, DOI 10.1016/j.ijhydene.2011.09.152.

(21)Bi, L.; Boulfrad, S.; Traversa, E. Steam electrolysis by solid oxide electrolysis cells (SOECs) with proton-conducting oxides. Chem. Soc. Rev. 2014, 43 (24), 8255-8270, DOI 10.1039/C4CS00194J.

(22)Chourashiya, G. M.; Urakawa, A. Solution combustion synthesis of highly dispersible and dispersed iridium oxide as an anode catalyst in PEM water electrolysis. J. Mater. Chem. A 2017, 5 (10), 4774-4778, DOI 10.1039/C6TA11047A.

(23)Fabbri, E.; Habereder, A.; Waltar, K.; Kötz, R.; Schmidt, T.J. Developments and perspectives of oxide-based catalysts for the oxygen evolution reaction. Catal. Sci. Tech. 2014, 4 (11), 3800-3821, DOI 10.1039/C4CY00669K.

(24)Chen, D.; Chen, C.; Baiyee, Z.M.; Shao, Z.; Ciucci, F. Nonstoichiometric Oxides as lowcost and highly-efficient oxygen reduction/evolution catalysts for low-temperature electrochemical devices. Chem. Rev. 2015, 115 (18), 9869-9921, DOI 10.1021/acs.chemrev.5b00073.

(25)Marcano D. C., Kosynkin D. V., Berlin J. M., Sinitskii A., Sun Z., Slesarev A., Alemany L. B., Lu W., Tour J. M. Improved synthesis of graphene oxide. ACS Nano 2010, 4 (8), 4806-4814, DOI 10.1021/nn1006368.

(26) Sun L., Fugetsu B. Mass production of graphene oxide from expanded graphite. Mater. Lett. 2013, 109, 207-210, DOI 10.1016/j.matlet.2013.07.072. 
(27)Peng L.; Xu Z.; Liu Z.; Wei Y.; Sun H.; Li Z.; Zhao X.; Gao C. An iron-based green approach to 1-h production of single-layer graphene oxide. Nat. Comms. 2015, 6, 5716, DOI 10.1038/ncomms6716.

(28)Liu, Y.; Liu, S.; Lai, X.; Miao, J.; He, D.; Li, N.; Luo, F.; Shi, Z.; Liu, S. PolyoxometalateModified sponge-like graphene oxide monolith with high proton-conducting performance. Adv. Funct. Mater. 2015, 25 (28), 4480-4485, DOI 10.1002/adfm.201501912.

(29)He G.; Chang C.; Xu M.; Hu S.; Li L.; Zhao J.; Li Z.; Li, Z.; Yin, Y.; Gang, M.; Wu, H.; Yang, X.; Guiver, M.D.; Jiang Z. Tunable Nanochannels along graphene oxide/polymer core-shell nanosheets to enhance proton conductivity. Adv. Funct. Mater. 2015, 25 (48), 7502-7511, DOI 10.1002/adfm.201503229.

(30)He, G.; Xu, M.; Zhao, J.; Jiang, S.; Wang, S.; Li, Z.; He, X.; Huang, T.; Cao, M.; Wu, H.; Guiver, M.D.; Jiang, Z. Bioinspired Ultrastrong solid electrolytes with fast proton conduction along 2D channels. Adv. Mater. 2017, 29 (28), 1605898, DOI 10.1002/adma.201605898.

(31)Wu, W.; Wang, J.; Liu, J.; Chen, P.; Zhang, H.; Huang, J. Intercalating ionic liquid in graphene oxide to create efficient and stable anhydrous proton transfer highways for polymer electrolyte membrane. Int. J. Hydro. Ener. 2017, 42 (16), 11400-11410, DOI 10.1016/j.ijhydene.2017.01.129.

\section{TOC GRAPHICS}




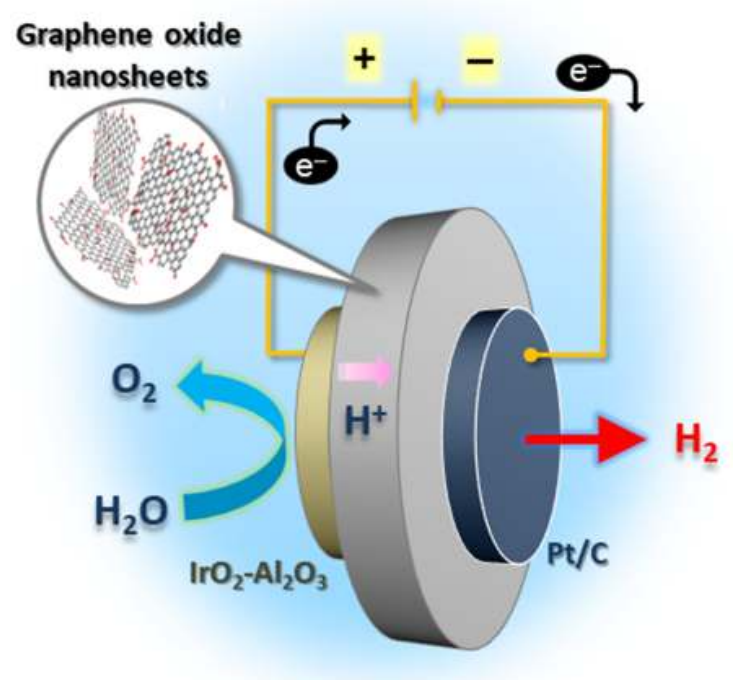

Synopsis. Proton-conducting carbon-based membranes were successfully used to electrochemically produce hydrogen from humid air.

p. 23 


\title{
Supporting Information
}

\section{Water vapor electrolysis with proton-conducting graphene oxide nanosheets}

\author{
Tetsuya Kida, ${ }^{* \dagger}$ Yuta Kuwaki,,$^{\dagger}$ Azumi Miyamoto, ${ }^{\dagger}$ Nur Laila Hamidah, ${ }^{\dagger}$ \\ Kazuto Hatakeyama, ${ }^{\dagger}$ Armando T. Quitain,,$^{\dagger}$ Mitsuru Sasaki, ${ }^{\dagger}$ Atsushi Urakawa ${ }^{\dagger}$
}

$\dagger$ Department of Applied Chemistry and Biochemistry, Kumamoto University,

Kurokami, Kumamoto, 860-8555, Japan

Institute of Chemical Research of Catalonia (ICIQ),

The Barcelona Institute of Science and Technology, Tarragona 43007, Spain

Corresponding Author: Tetsuya Kida

E-mail: tetsuya@kumamoto-u.ac.jp

p. 24 
TEL: +81-96-342-3664, FAX: +81-96-342-3679

The supporting information contains:

Number of pages: 5

Number of figures: 7

p. 25 

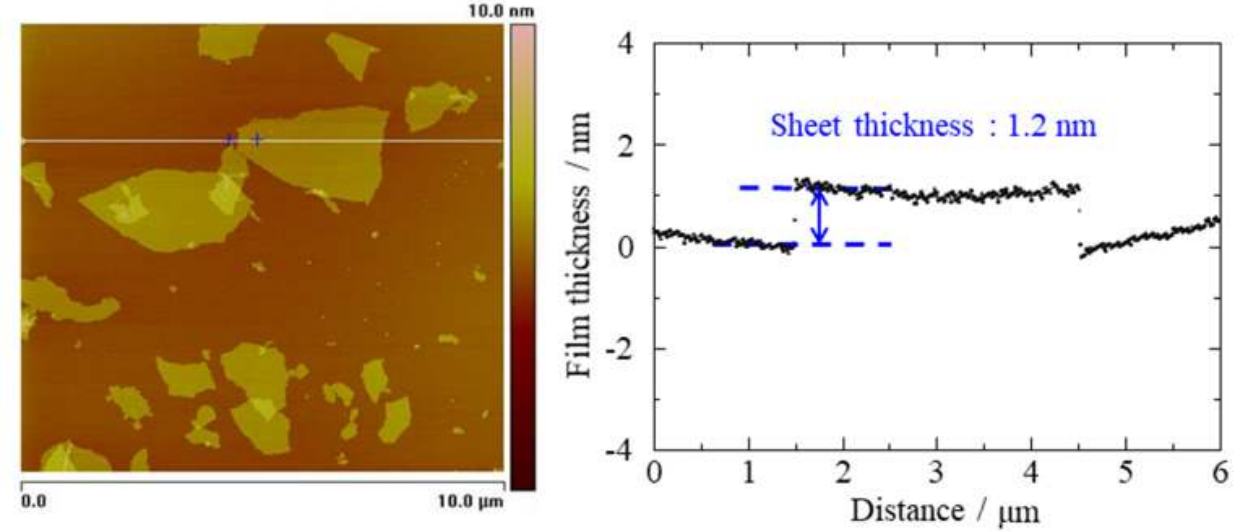

Figure S1. Representative atomic force microscopy image of graphene oxide (GO) nanosheets.

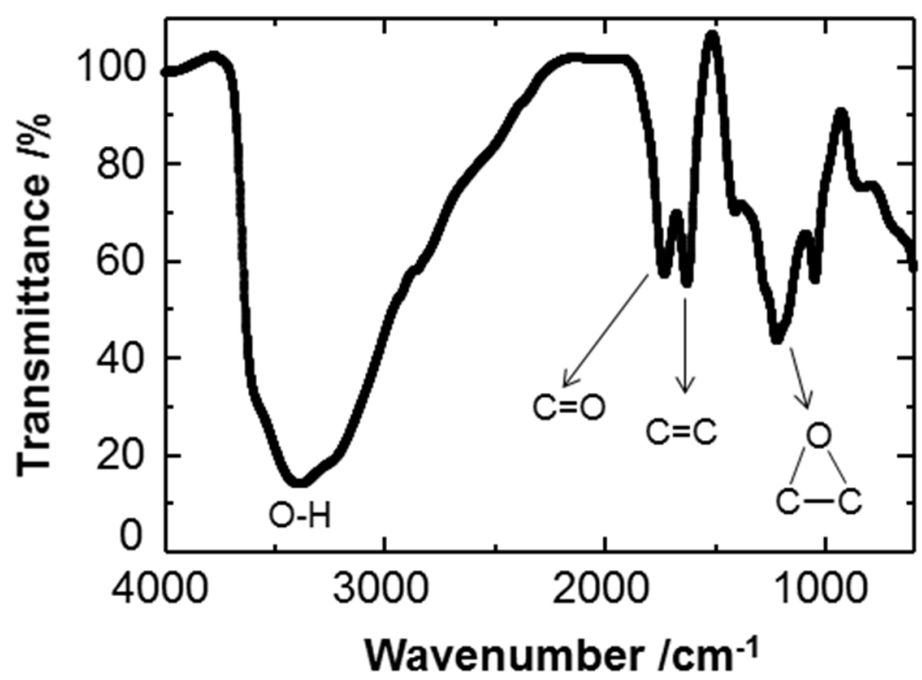

Figure S2. Representative Fourier transform infrared spectrum of a GO membrane. 


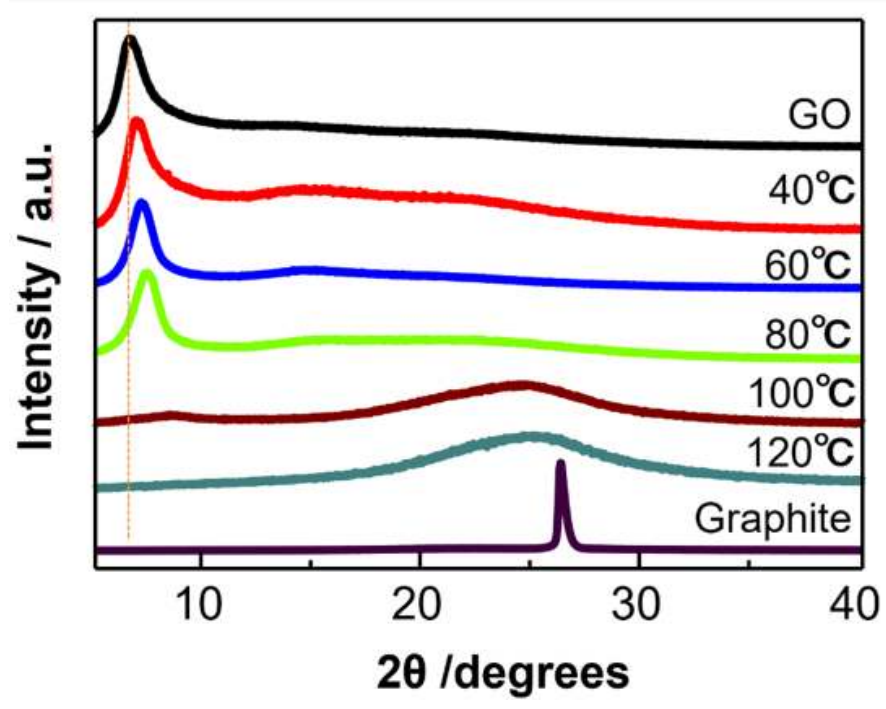

Figure S3. XRD patterns of a GO membrane after annealing at $40-120^{\circ} \mathrm{C}$, together with that for before annealing. The XRD pattern of graphite is also shown for comparison.

Table S1. Interlayer distance of a GO membrane after annealing at $40-120^{\circ} \mathrm{C}$, together with that for before annealing.

\begin{tabular}{ccc} 
Sample & $\begin{array}{c}\text { Annealing temperature / } \\
{ }^{\circ} \mathbf{C}\end{array}$ & Interlayer distance / $\mathbf{\AA}$ \\
\hline GO & - & 13.32 \\
GO & 40 & 12.65 \\
GO & 60 & 12.40 \\
GO & 80 & 12.11
\end{tabular}

p. 27 
(a)

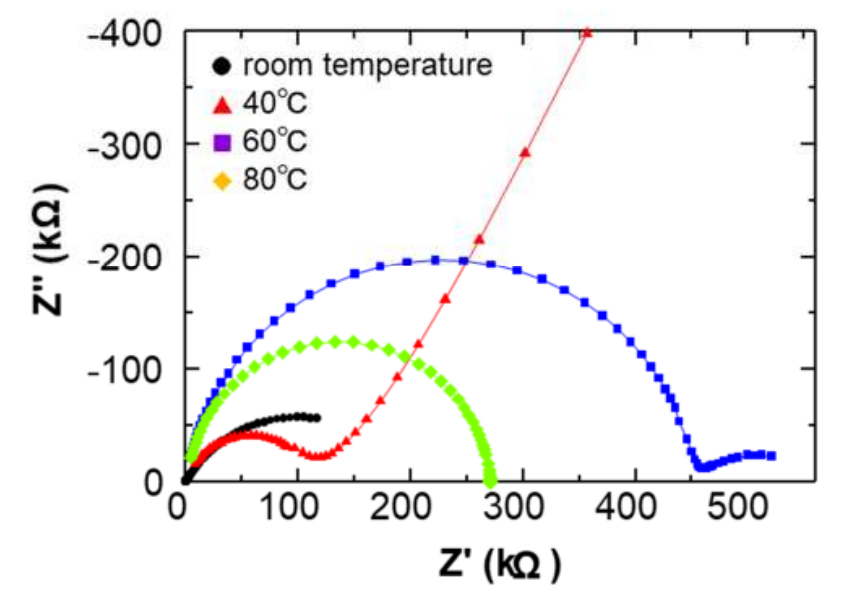

(b)

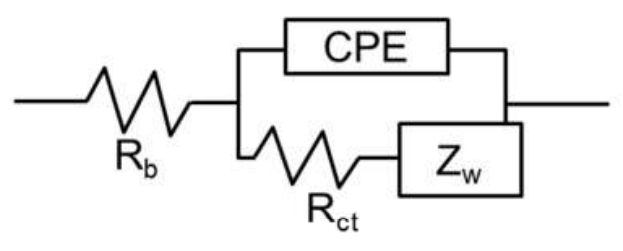

$\mathrm{R}_{\mathrm{b}}$ : Bulk Resistance CPE: Constant Phase Element $\mathrm{R}_{\mathrm{ct}}$ : Charge Transfer Resistance $\mathrm{Z}_{\mathrm{w}}$ : Warburg Element

Figure S4. (a) Cole-Cole plots of the complex impedance of the GO membrane in humid air $(35 \% \mathrm{RH})$ at different temperatures. (b) Equivalent circuit to estimate the bulk resistance in the GO membrane. For impedance measurements, Pt electrodes were formed by sputtering, and then the membrane was sandwiched with two Pt meshes as electron collectors. 


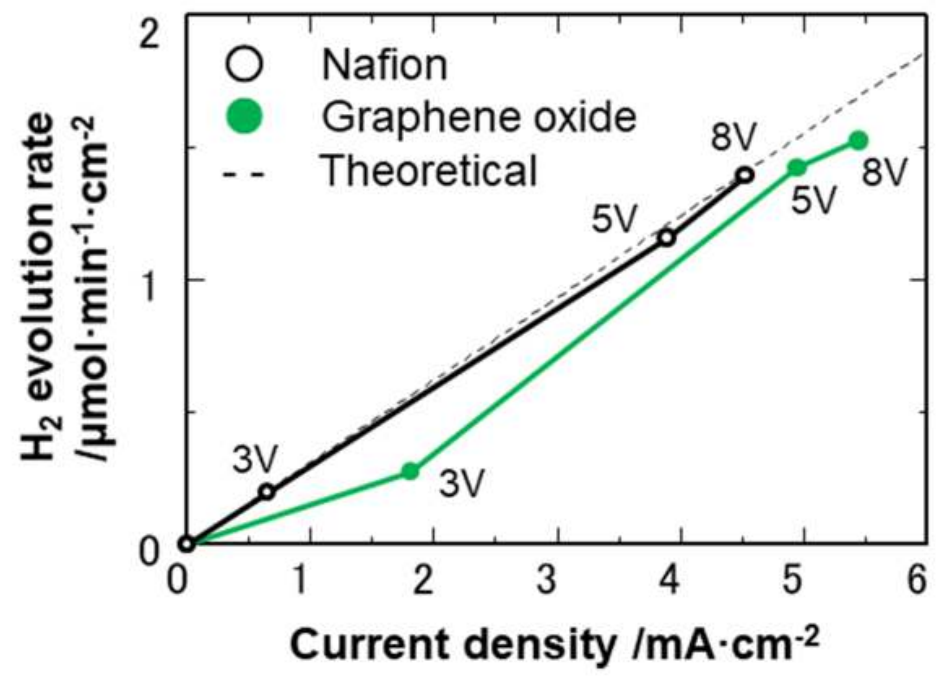

Figure S5. $\mathrm{H}_{2}$ evolution rate as a function of current density for the $\mathrm{GO}$ device that is sandwiched by two Pt mesh electrodes. Electrolysis of water vapor was performed at room temperature. For comparison, a Nafion membrane fitted with two Pt mesh electrodes was tested for its performance in water vapor electrolysis.

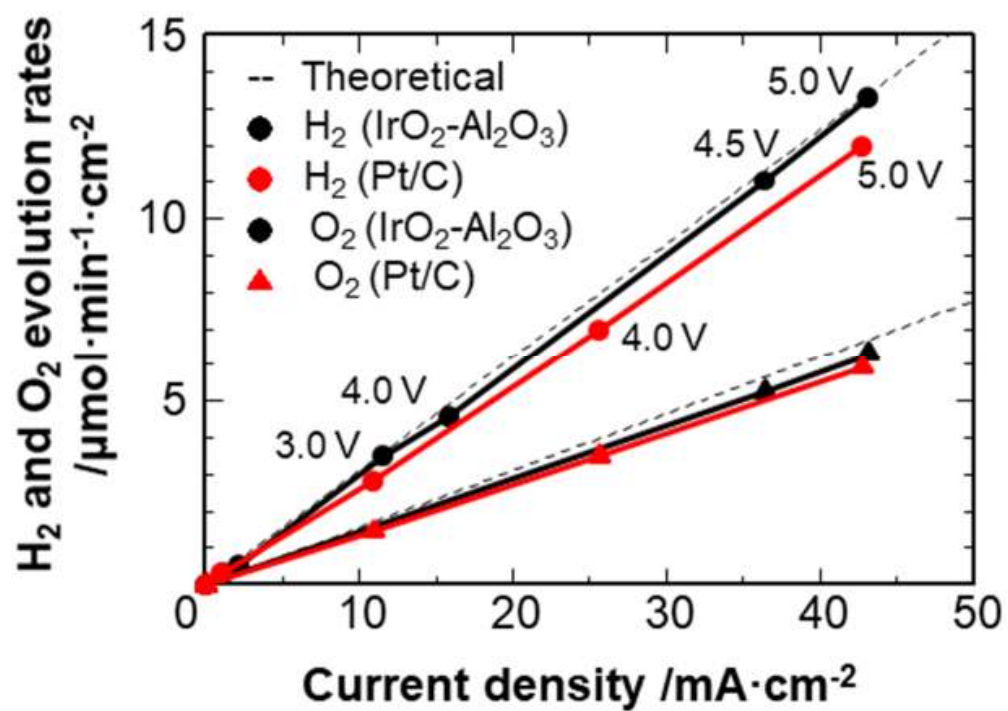

Figure S6. Hydrogen and oxygen evolution rates against current density in water vapor electrolysis at room temperature for devices using $\mathrm{IrO}_{2}-\mathrm{Al}_{2} \mathrm{O}_{3}$ or $\mathrm{Pt} / \mathrm{C}$ in the anode. 


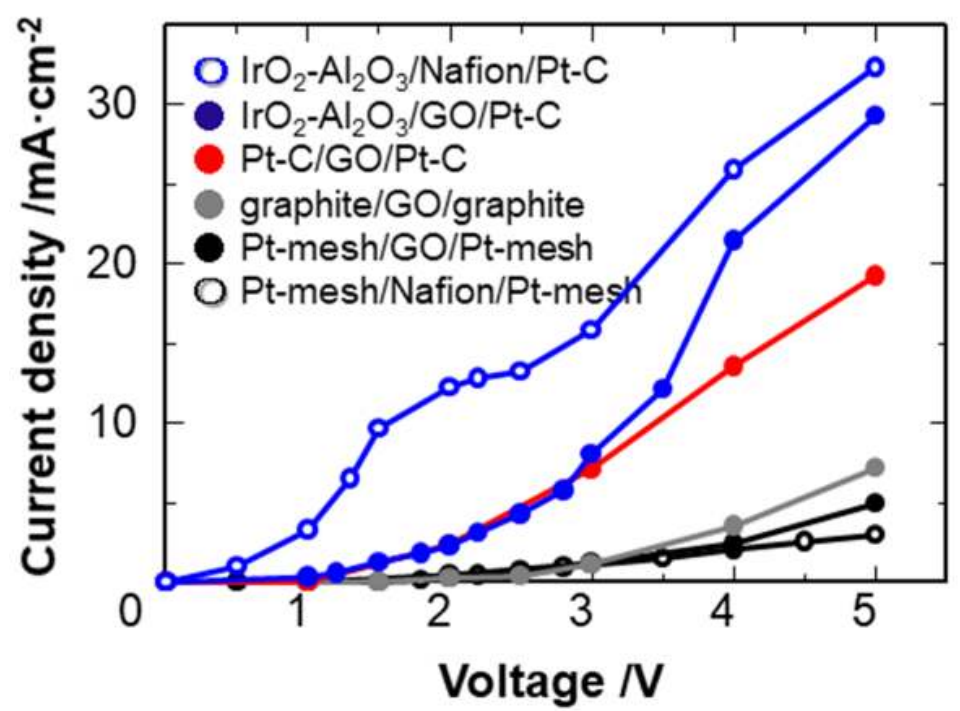

Figure S7. Dependence of current density on applied voltage for GO devices fitted with different electrocatalysts at room temperature. The device structure is expressed as anode/membrane/cathode. 